\title{
ARTÍCULOS
}

\section{Tendencias sucesionales de los bosques montanos subtropicales del noroeste argentino}

\author{
Successional patterns of subtropical montane forest from northwestern Argentina
}

\author{
Julieta Carilla $^{\text {a*, }}$ Ricardo Grau ${ }^{\text {a }}$ \\ *Autor de correspondencia: ${ }^{a}$ CONICET, Universidad Nacional de Tucumán, Instituto de Ecología Regional, \\ cc 34 (4107) Yerba Buena, Tucumán, Argentina, julietacarilla@yahoo.com.ar
}

\begin{abstract}
SUMMARY
Subtropical montane forests from NW Argentina are characterized by a mosaic of different forest unities originated after grazing pressure reduction in different successional stages. This study analyzed successional trajectory of four forest situations, in terms of composition, structure and demography; including three secondary forest situations dominated by Alnus acuminata, Crinodendron tucumanum and Podocarpus parlatorei, and one old grow forests situation dominated by Myrtaceae family species. Ten permanent plots established at 1,700 m asl in Sierra San Javier, Tucumán, were permanently monitored in 1991 and re-measured in 1996, 2001 and 2007. Alnus acuminata and C. tucumanum secondary forests presented a fast accumulation of biomass, reaching $181 \mathrm{t} / \mathrm{ha}$ in few decades. $P$. parlatorei forests reached high values of biomass (393 t/ha), similar to those reached by Myrtaceae forest (418 $\mathrm{t} / \mathrm{ha}$ ). However, in both situations, forest biomass decreased due to the mortality of pioneer species that accumulated great amount of basal area and to the low recruitment of old growth forest species. We conclude that despite variability in change rates, secondary forest tended to converge in composition towards more diverse Myrtaceae forest with slow replacement of old growth species and high understory treelet recruitment, as Solanum grossum. These long term studies are useful to understand montane forest dynamic and their ecosystems services, such as their effect in carbon sequestration, watershed protection and regional biodiversity conservation.
\end{abstract}

Key words: biomass, permanent plots, Podocarpus parlatorei, secondary succession, demographic rates.

\section{RESUMEN}

Los bosques montanos subtropicales del noroeste argentino forman un mosaico de diferentes unidades boscosas originadas por la reducción de la presión ganadera en distintas etapas sucesionales. Este estudio analizó la trayectoria sucesional de cuatro situaciones boscosas con diferente composición, estructura y demografía, incluyendo tres situaciones de bosques secundarios dominados por Alnus acuminata, Crinodendron tucumanum y Podocarpus parlatorei, y una de bosques maduros dominados por mirtáceas. Se monitorearon 10 parcelas permanentes establecidas en 1991 en la sierra de San Javier, Tucumán a 1.700 m s.n.m., y remedidas en 1996, 2001 y 2007. Los bosques secundarios de A. acuminata y C. tucumanum acumularon biomasa rápidamente, alcanzando 181 t/ha en pocas décadas. Los bosques de $P$. parlatorei alcanzaron valores elevados de biomasa (393 t/ha), similar a los bosques maduros de mirtáceas (418 t/ha). En ambas situaciones disminuyó la biomasa debido a la muerte de especies pioneras que acumularon gran cantidad de área basal y al reducido reclutamiento de especies arbóreas sucesionalmente tardías. Se concluyó que, a pesar de la variabilidad en las tasas de cambio, los bosques secundarios tendieron a converger composicionalmente hacia bosques de mirtáceas, con un lento reemplazo de especies de bosque maduro y un abundante reclutamiento de especies de sotobosque, destacándose Solanum grossum. Estos estudios a largo plazo son útiles para entender la dinámica de los bosques de montaña y sus servicios ecosistémicos, como su efecto sobre la fijación de carbono, protección de cuencas fluviales y conservación de la biodiversidad regional.

Palabras clave: biomasa, parcelas permanentes, Podocarpus parlatorei, sucesión secundaria, tasas demográficas.

\section{INTRODUCCIÓN}

En muchas regiones montañosas tropicales y subtropicales, tierras forestales marginales previamente usadas para actividades agrícolas y ganaderas están siendo abandonadas como consecuencia de diferentes procesos socioeconómicos (Aide y Grau 2004, Clark et al. 2009). Esto, sumado a decisiones de manejo que excluyen áreas de uso productivo para fines conservacionistas, represen- tan una oportunidad para la recuperación de formaciones boscosas que proveen servicios ecosistémicos importantes como la protección de cuencas (Grau et al. 2008), producción de madera (Guariguata y Ostertag 2001), recuperación de la biodiversidad local (Grau et al. 2007), conectividad de fragmentos de bosque (Chazdon 2003) y, en forma creciente, captación de carbono atmosférico en forma de biomasa (Silver et al. 2001, Grau et al. 2004).

Este es el caso de los bosques subtropicales de mon- 
taña del noroeste argentino o yungas, que tienen gran importancia ecológica regional principalmente por su alta biodiversidad y por su rol en la regulación de las principales cuencas hídricas (Huzinger 1997). En sincronía con la reducción regional del pastoreo, el piso montano superior de las yungas argentinas muestra procesos de expansión de bosques (Grau 1985). Entre los 1.600 y 2.000 m s.n.m. esta dinámica tiene como resultado un mosaico de pastizales, arbustales y bosques de distinta edad sucesional dominados por diferentes especies pioneras como Podocarpus parlatorei Pilg. y Alnus acuminata H.B.K. (Cabrera 1976, Arturi et al. 1998, Brown et al. 2001).

La sierra de San Javier representa uno de los sitios mejor estudiados dentro de esta ecoregión. Estudios basados en sensores remotos muestran que durante las últimas décadas, la cobertura forestal de la sierra aumentó (Grau et al. 2008). El mosaico de fisonomías boscosas del bosque montano superior fue bien descrito a principios de los años 90 (Arturi et al.1998, Carilla et al. 2006) cuando se establecieron parcelas permanentes que son utilizadas en el presente análisis. El monitoreo de parcelas permanentes permite evaluar los cambios florísticos y estructurales del bosque de manera precisa, y cuantificar las tasas de recuperación durante procesos sucesionales. La mayoría de los estudios sobre dinámica de bosques neotropicales basados en parcelas permanentes se desarrollan en bosques húmedos de tierras bajas (Phillips y Gentry 1994, Phillips et al. 1994, Condit 1995, Condit et al.1995, Finegan y Delgado 2000). Este sistema de parcelas, representativo de distintas unidades fisonómicas y con 16 años de datos de cambio, contribuye a expandir los conocimientos de dinámica en bosques de montaña, comparativamente mucho menos estudiados; y representa el estudio basado en parcelas permanentes de más longitud temporal en esta ecoregión.

La hipótesis que se puso a prueba es que las tendencias sucesionales de composición, estructura y demografía están influidas por la historia de uso del suelo y de colonización temprana por distintas especies; y que bosques secundarios de distintos orígenes y composición tienden a converger sucesionalmente hacia la composición del bosque maduro. Para esto, se plantearon los siguientes objetivos específicos: 1) analizar los patrones temporales de composición, demografía y estructura de parcelas de bosque secundarios y maduros representativos del bosque montano superior de las yungas; 2) estimar la biomasa acumulada por estos bosques y su importancia relativa en el balance de carbono; y 3) analizar, en detalle, la dinámica poblacional de la especie dominante, Podocarpus parlatorei, y su importancia en la dinámica del bosque.

\section{MÉTODOS}

Área de estudio. Los bosques estudiados están ubicados en el Parque Sierra San Javier ( $26^{\circ} 47^{\prime}$ S y $65^{\circ} 22^{\prime}$ O), área protegida desde el año 1973, perteneciente a la Universidad Nacional de Tucumán, Argentina (figura 1). La sierra está situada a $13 \mathrm{~km}$ del Gran San Miguel de Tucumán (cerca de un millón de habitantes) y, a pesar del elevado índice de urbanización registrado en los últimos 30 años, también se registró un incremento en el área boscosa (Grau et al. 2008). Antes de la creación del parque, el principal uso de la tierra era el pastoreo (ovino y vacuno) y su gradual eliminación resultó en un mosaico de pastizales, arbustales y bosques con diferentes edades de abandono (Grau 1985, Moyano y Movia 1989, Arturi et al. 1998, Grau et al. 2010). Actualmente, el manejo del bosque se limita a la exclusión del ganado y la supresión del fuego. La zona donde se establecieron las parcelas está alejada de la influencia antrópica directa y son infrecuentes disturbios de grandes escalas que afectan la vegetación.

El clima es de tipo subtropical con estación seca, las precipitaciones medias anuales para el área de estudio son $1.500 \mathrm{~mm}$ aproximadamente. Las precipitaciones son principalmente estivales; en invierno vientos húmedos del sur y este, condensan la humedad al chocar contra las laderas, originando precipitación horizontal, correspondiente al $20 \%$ de las precipitaciones totales (Huzinger 1997) y ocasionalmente nieva. La temperatura promedio anual es de $14{ }^{\circ} \mathrm{C}$, con máximas cercanas a $30{ }^{\circ} \mathrm{C}$ (diciembre - enero) y mínimas bajo 0 (julio - agosto) y con heladas frecuentes en invierno. Desde la década del 50, el noroeste argentino ha experimentado un aumento de las precipitaciones estivales del 20 \% aproximadamente (Minetti y Vargas 1997, Villalba et al. 1998) asociado a cambios en los patrones de circulación atmosférica (Labraga 1997).

La vegetación corresponde al bosque montano superior de las yungas argentinas (Cabrera y Willink 1980). Las especies arbóreas características de estos bosques son Alnus acuminata, Crinodendron tucumanum Lillo y Podocarpus parlatorei en estadios tempranos - intermedios de sucesión, e Ilex argentina Lillo, Prunus tucumanensis Lillo, Cedrela lilloi C. DC., Juglans australis Griseb y especies de la familia Myrtaceae en etapas sucesionales tardías y bosques maduros (Grau et al. 2010). Los arbustales están dominados por Baccharis articulata (Lam.) Pers., B. tucumanensis Hook. et Arn. (Asteraceae) y Chusquea lorentziana Griseb. (Bambuceae) y los pastizales por Festuca hieronymi Hack., Deyeuxia polygama (Griseb.) Parodi y Stipa eriostachya Kunth (Poaceae) (Giusti et al. 1997).

En 1991 se establecieron 10 parcelas permanentes en diferentes situaciones boscosas: dos dominados por $A$. acuminata, dos por C. tucumanum, cuatro por P. parlatorei y dos bosques maduros dominados por especies de la familia Myrtaceae. El tamaño de las parcelas fue de 0,12 a 0,48 ha, organizadas en cuadrantes contiguos de $400 \mathrm{~m}^{2}$ (20x20 m, de 4 a 12 cuadrantes), abarcando un área total de 2,68 ha (cuadro 1). En cada parcela se identificaron todos los árboles, marcados con chapas numeradas y mapeados en sistema de coordenadas X-Y. Se midió el diámetro a la altura del pecho (DAP) de todos los árboles vivos $\geq 10$ $\mathrm{cm}$. Las parcelas fueron remedidas en diciembre de 1996, 2001 y 2007 (5, 10 y 16 años desde su establecimiento). 


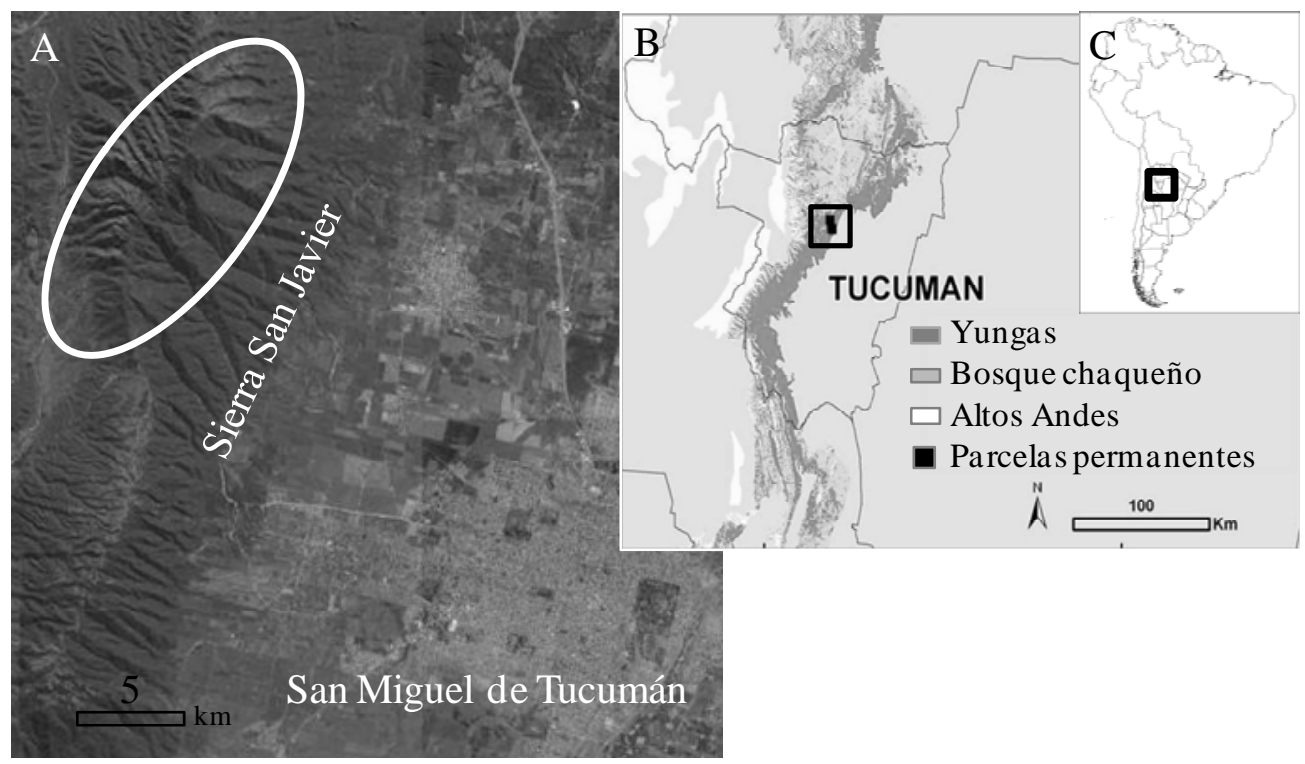

Figura 1. Área de estudio. A) Imagen Spot de la zona de San Javier, Tucumán. El oval blanco indica la ubicación de las parcelas. B) Mapa de la provincia de Tucumán (cartografía: SIGA ProYungas), el cuadrado negro indica la posición de la figura A. B) Mapa de América del Sur, el cuadrado negro indica la ubicación de la figura B.

Study area. A) Spot image of San Javier, Tucumán. The white oval delimits permanent plot localization. B) Map of Tucumán Province (Cartography SIGA ProYungas), the black square indicates figure A location. C) Map of South America, the black square indicates figure B location.

Más información sobre las parcelas permanentes se puede encontrar en Arturi et al. (1998) y Carilla et al. (2006).

Se incluye un análisis detallado de la sucesión secundaria de $P$. parlatorei a nivel poblacional, enfocado en la estructura de tamaños, biomasa aérea y crecimiento anual, dada su importancia relativa en la dinámica del bosque. Podocarpus parlatorei es una especie pionera que se establece en los primeros estadios de sucesión y persiste hasta etapas sucesionales intermedias - tardías, coexistiendo con especies típicas del bosque maduro. Su longevidad y tolerancia limitada a la sombra le confiriere características de especie pionera-longeva. Es dispersada por aves y su establecimiento es favorecido por la presencia de arbustos y bosques abiertos de A. acuminata (Grau 2006).

La edad de los bosques fue estimada por Carilla et al. (2006) basada en técnicas dendrocronológicas para los bosques de A. acuminata y P. parlatorei, complementando con relaciones diámetro/crecimiento, también usadas para los bosques de $C$. tucumanum y mirtáceas. Las muestras fueron tomadas con barreno de incremento de los individuos más grandes de cada bosque, montadas, pulidas y contados los anillos de crecimiento. Se promedió la edad estimada de los árboles más viejos por bosque; las edades variaron desde 45 años para el bosque más joven (de A. acuminata joven) a más de 500 años para los bosques maduros de mirtáceas (cuadro 1).

Análisis de datos. Con el propósito de explorar las relaciones florísticas y tendencias composicionales de las especies estructuralmente dominantes, se calculó el índice de distancia de Bray Curtis (Legendre y Legendre 1998). Posteriormente se hizo un ordenamiento de "escalamiento multidimensional no-métrico" (NMDS) para reducir la dimensionalidad de la matriz de similitud (Kruskal y Wish 1978). Las trayectorias de cambio en la composición de especies se indicaron mediante "vectores sucesionales" representados por flechas. El escalamiento multidimensional no-métrico es una de las técnicas de ordenamiento más usadas para comunidades, porque es robusto e independiente a supuestos sobre distribución de especies a lo largo de los gradientes subyacentes al ordenamiento (McCune y Grace 2002). La matriz de similitud entre parcelas en 1991, 1996, 2001 y 2007 se basó en la composición de "especies de dosel", definidas como aquellas que en condiciones características de buen desarrollo superan los $12 \mathrm{~m}$ (cuadro 2). Para las corridas de escalamiento multidimensional no-métrico se usaron las coordenadas originadas de una corrida con modo autopiloto en PC-ord 5.0 (McCune y Mefford 1999), con la opción lento y completo (corridas reales $=40$ e iteraciones máximas $=400$ ). Los ejes del ordenamiento se rotaron para lograr la dirección de la mayor variación con el eje horizontal (varimax rotation). El porcentaje de la variación de los datos originales explicado por el ordenamiento se calculó con correlaciones Pearson $r$ entre los valores de los ejes en el espacio de ordenamiento y la distancia en la matriz original (McCune y Grace 2002). El estrés final del ordenamiento para la configuración de dos dimensiones fue menor a 20 , lo que indica un ajuste relativamente bueno entre la configuración del gráfico y la matriz de Bray Curtis, y fue signifi- 


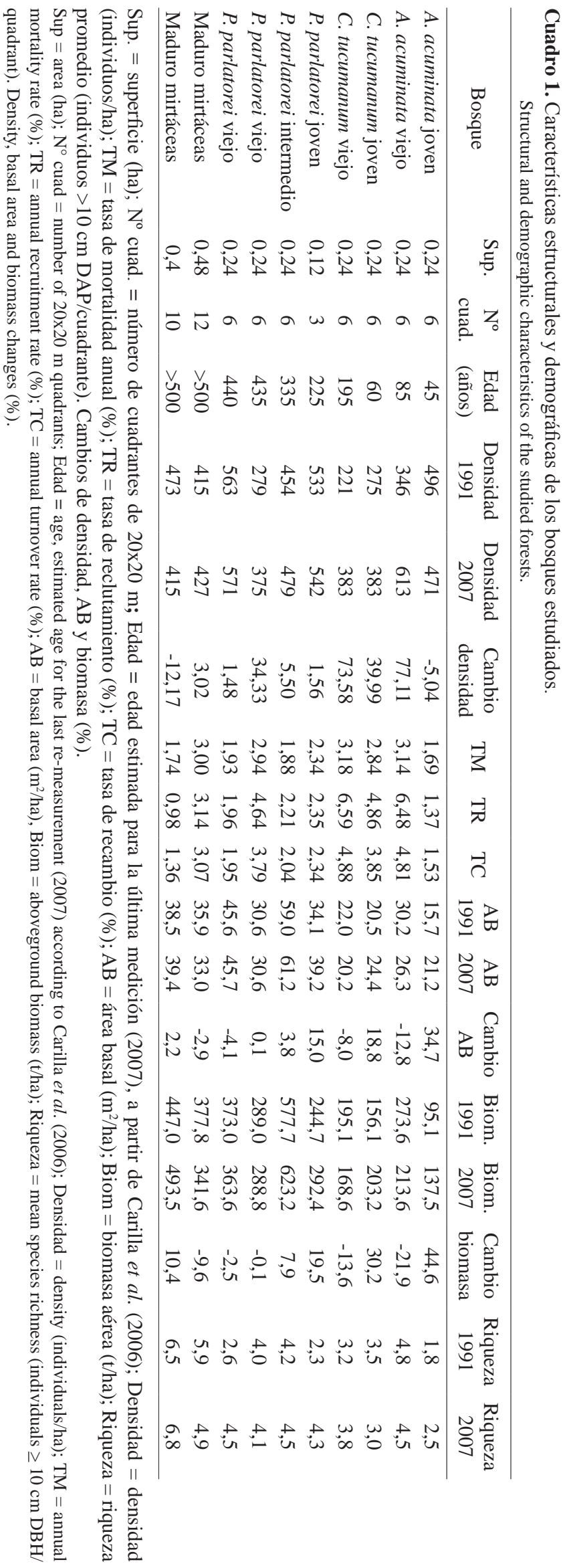


Cuadro 2. Lista de especies, familias botánicas y tipo de árbol según la altura alcanzada por el adulto. Species list, botanical families and tree type according to adult height.

\begin{tabular}{|c|c|c|c|c|}
\hline Especie & Familia & $\mathrm{D}$ o $\mathrm{S}$ & $\begin{array}{l}\text { Densidad de } \\
\text { leño }\left(\mathrm{g} / \mathrm{cm}^{3}\right)\end{array}$ & Referencias \\
\hline Allophylus edulis (St. Hill) Radlk & Sapindaceae & S & 0,708 & Easdale (2006) \\
\hline Blepharocalyx salicifolious (H.B.K.) O. Berg & Myrtaceae & $\mathrm{D}$ & 0,768 & \\
\hline Cedrela lilloi C. DC. & Meliaceae & $\mathrm{D}$ & 0,406 & \\
\hline Duranta serratifolia (Griseb.) Kuntze & Verbenaceae & $\mathrm{D}$ & 0,562 & \\
\hline Juglans australis Griseb & Juglandaceae & $\mathrm{D}$ & 0,490 & \\
\hline Piper tucumanum C. DC. & Piperaceae & $\mathrm{S}$ & 0,457 & \\
\hline Vassovia breviflora (Sendnt.) Hunz. & Solanaceae & $\mathrm{S}$ & 0,547 & \\
\hline Alnus acuminata H.B.K. & Betulaceae & $\mathrm{D}$ & 0,429 & Easdale et al. (2007) \\
\hline Crinodendron tucumanum Lillo & Elaeocarpaceae & $\mathrm{D}$ & 0,458 & \\
\hline Ilex argentina Lillo & Aquifoliaceae & $\mathrm{D}$ & 0,500 & \\
\hline Myrcianthes mato (Griseb.) McVaugh & Myrtaceae & $\mathrm{D}$ & 0,695 & \\
\hline Myrcianthes pseudomato (D. Legrand) McVaugh & Myrtaceae & $\mathrm{D}$ & 0,727 & \\
\hline Myrcianthes callicoma Mc Vaugh & Myrtaceae & $\mathrm{D}$ & $0,711 *$ & \\
\hline Podocarpus parlatorei Pilg. & Podocarpaceae & $\mathrm{D}$ & 0,440 & \\
\hline Prunus tucumanensis Lillo & Rosaceae & $\mathrm{D}$ & 0,609 & \\
\hline Prunus persica (L.) Batsch & Rosaceae & $\mathrm{D}$ & $0,609 *$ & \\
\hline Sambucus nigra L. & Caprifoliaceae & $\mathrm{D}$ & $0,429 *$ & \\
\hline Kaunia lasiophtalmun Griseb. & Asteraceae & $\mathrm{S}$ & $0,494 *$ & \\
\hline Solanum grossum C.V. Morton & Solanaceae & S & 0,494 & \\
\hline Azara salicifolia Griseb. & Flacourtiaceae & $\mathrm{S}$ & $0,640 *$ & Chave et al. (2006) \\
\hline Dunalia lorentzii (Damner) Sleumer & Solanaceae & $\mathrm{D}$ & $0,510^{*}$ & \\
\hline
\end{tabular}

$\mathrm{D}$ = dosel, altura promedio de adulto $>12 \mathrm{~m}$, cálculo estimado para cada especie y no varía según la edad del bosque. S = sotobosque, densidades de leño seco y referencia bibliográfica. *No hay datos de densidad disponibles y fue calculada teniendo en cuenta los criterios descritos en el texto. Nomenclatura botánica de Zuloaga et al. (2008).

$\mathrm{D}=$ canopy species, adult mean height $>12 \mathrm{~m}$, estimated for each species and does not change with forest age. $\mathrm{S}=$ understory species, dry wood density, references. * No density data available, calculated according to criteria mentioned in text. Botanical nomenclature from Zuloaga et al. (2008).

cativamente diferente al azar (Montecarlo: 250 corridas, $P=0,004)$. Para cuantificar las similitudes/disimilitudes entre los bosques, reflejadas en el ordenamiento y asociarlas con tendencias sucesionales, se compararon los índices de distancia Bray Curtis calculados para 1991 y 2007 con valores de 0 (máxima similitud) a 1 (mínima similitud).

Para describir como las especies influyeron en la segregación de los bosques en el diagrama de ordenamiento, se calcularon sus posiciones promedio a lo largo de cada eje mediante método de "pesado promedio" (McCune y Grace 2002). Para evaluar la significancia estadística de las especies en la segregación de los bosques en el ordenamiento se hicieron correlaciones de Kendall (Sokal y Rohlf 1995) entre la abundancia de especies y los valores de los bosques sobre los ejes.

Para evaluar las diferencias en la riqueza de especies, entre los bosques y entre 1991 y 2007, se usó un ANDEVA de dos vías, usando riqueza como variable respuesta y los diferentes tipos de bosques y años como variables categóricas. Se verificó que los datos cumplan con el supuesto de normalidad.

Para estudiar los patrones demográficos y estructurales, se calculó la densidad total de árboles (individuos/ ha), riqueza de especies (número de especies promedio por cuadrante), tasas de mortalidad [1], reclutamiento [2], y recambio (\% anual), tasa de crecimiento anual y área basal $\left(\mathrm{m}^{2} / \mathrm{ha}\right)$. Las tasas de mortalidad y reclutamiento se calcularon a partir de la ecuación de Sheil et al. (1995) y Bellingham y Sparrow (2009) que tiene en cuenta el periodo entre dos mediciones.

$$
\begin{aligned}
& \mathrm{M}=1-\left[1-\left(\mathrm{N}_{0}-\mathrm{N}_{1}\right) / \mathrm{N}_{0}\right]^{1 / \mathrm{t} *} 100 \\
& \mathrm{R}=1-\left(1-\mathrm{N}_{\mathrm{r}}-\mathrm{N}_{1}\right)^{1 / \mathrm{t} * 100}
\end{aligned}
$$

Donde $\mathrm{M}$ = tasa de mortalidad; $\mathrm{N}_{0}=$ número de individuos en la primera medición; $N_{1}=$ número de sobrevivientes en la siguiente medición; $\mathrm{t}=$ período entre dos monitoreos; $\mathrm{R}$ = tasa de reclutamiento; $\mathrm{N}_{\mathrm{r}}=$ número de individuos reclutados entre dos períodos.

La tasa de crecimiento anual por especie se calculó para el período entre dos monitoreos (5 - 6 años), considerando individuos vivos entre 10 y $40 \mathrm{~cm}$ de DAP, para que sea comparable entre especies y evitar estimaciones erróneas debido a la edad del árbol (tamaño). Para los individuos multifustales, la tasa de crecimiento anual se estimó a base del cálculo de un único diámetro virtual, transformando nuevamente el área basal (suma del área basal de todos los fustes de un árbol) a DAP (Easdale et al. 2007).

La biomasa aérea (t/ha) se calculó a partir de Chave et al. (2005) [3] para bosques húmedos, que tiene en cuenta el diámetro del árbol y la densidad del leño: 
AGB $=\mathrm{p}^{*} \exp (-1,499+2,148 \ln \mathrm{DAP})+0,207(\ln \mathrm{DAP})^{2}-$ $\left.0,0281(\operatorname{lnDAP})^{3}\right)$

Donde AGB = biomasa aérea ( $\mathrm{t} / \mathrm{ha}$ ); $\mathrm{p}=$ densidad de leño $\left(\mathrm{g} / \mathrm{cm}^{3}\right) ; \mathrm{DAP}=$ diámetro a la altura del pecho $(\mathrm{cm})$.

La ecuación [3] es válida para un amplio rango de tamaños de árboles (DAP: 5 a $156 \mathrm{~cm}$ ) y para todas las angiospermas (Chave et al. 2005). En este estudio fue usada también para la gimnosperma $P$. parlatorei dado que a simple vista su arquitectura y porte no es el característico de una conífera, sino que presenta patrones de ramificación semejantes a los de otras especies arbóreas.

Los datos de densidad de leño se obtuvieron de fuentes bibliográficas (cuadro 2). Para las especies sin información disponible se usó el de otras especies congenéricas presentes en la Sierra San Javier: para Prunus persica (L.) Batsch se usó la densidad de P. tucumanensis, para Kaunia lasiophtalmun Griseb. la de K. saltensis (Hieron.) R. M. King et H. Rob. (único dato de densidad para este género). Para Myrcianthes callicoma Mc Vaugh se promedió la densidad de Myrcianthes mato (Griseb.) McVaugh y Myrcianthes pseudomato (D. Legrand) McVaugh. Para Solanum grossum se usó la densidad de Solanum riparium Pers. presente en bosques más bajos (a 1.000 m. s.n.m.). Para Dunalia lorentzii (Damner) Sleumer y Azara salicifolia Griseb. se aplicaron las densidades promedio de las respectivas familias Solanaceae y Flacourtiaceae (cuadro 2).

Los resultados se reportan enfocados en $P$. parlatorei en histogramas de distribución de tamaño por bosque a partir del DAP virtual. Un $15 \%$ de individuos de P. parlatorei presentó dos fustes y un $5 \%$ tres fustes.

\section{RESULTADOS}

Densidad, composición y riqueza (1991- 2007). Se registró un promedio de 456 individuos arbóreos $\geqq 10 \mathrm{~cm}$ por hectárea en 2007 (1.222 árboles en 2,68 ha). Fueron encontradas 22 especies, de las cuales 13 corresponden al dosel arbóreo y nueve al sotobosque (cuadro 2, apéndice 1). El bosque viejo de A. acumuinata presentó la mayor densidad (613 árboles/ha), seguido por el bosque de $P$. parlatorei viejo-2 y el joven con 571 y 542 árboles/ha, respectivamente. Los máximos incrementos en densidad se registraron en los bosques viejos de A. acuminata y $C$. tucumanum con un aumento del 77 y $74 \%$ y la mayor disminución se registró en el bosque de mirtáceas-2 con un $12 \%$ (cuadro 1 ).

En el ordenamiento multidimensional no-métrico, los dos primeros ejes explicaron el $79 \%$ de la varianza (eje 1 : $47 \%$ y eje 2: $32 \%$ ). A lo largo del eje 1 se identificaron dos grandes grupos: 1 ) en el sector positivo, bosques de $P$. parlatorei y mirtáceas y 2) en el sector negativo, bosques de C. tucumanum y A. acuminata. Dentro del grupo 1 (sector positivo del eje 1) se pueden distinguir dos subgrupos: 1.1) bosques de P. parlatorei (joven, intermedio y viejo-2) en la porción positiva del eje 2 y 1.2) bosques maduros de mirtáceas y de $P$. parlatorei viejo-1 en la porción negativa del eje 2 (figura 2). En general, se observó que las trayectorias sucesionales (cambios en el espacio de ordenación entre 1991 y 2007), tendieron a dirigirse hacia el extremo inferior derecho del ordenamiento, donde están ubicados los bosques de mirtáceas. El bosque joven de $C$. tucumanum muestra una trayectoria relativamente diferente orientada hacia la parte positiva del eje 1. Los bosques de mirtáceas y el bosque viejo-1 de $P$. parlatorei (extremo inferior derecho), mostraron pocos cambios temporales composicionales, reflejados en la corta longitud de las flechas. El bosque joven de A. acuminata fue el más diferente de todas las situaciones ubicándose en la parte negativa del eje 2 .

Las correlaciones de Kendall entre los valores de ambos ejes y la abundancia de las especies mostraron 20 coeficientes de correlación significativos $(P<0,001$; cuadro 3$)$.

Los cambios en el índice de distancia Bray Curtis sugiere una tendencia en que los bosques secundarios tienen una trayectoria hacia bosques de mirtáceas (valores menores del índice en 2007 que en 1991). Este patrón se observó en la mayoría de los bosques, excepto en los de P. parlatorei intermedio y de P. parlatorei viejo-1, donde el índice no varió (figura 3 ).

La riqueza de los bosques de mirtáceas (14 especies en 1991 y 2007) y de los bosques de P. parlatorei (11 especies en 1991 y 15 en 2007) fue significativamente mayor que la de los bosques de A. acuminata - C. tucumanum (seis especies en 1991 y nueve en 2007) (ANDEVA: $F(2,128)$ $=44,7, P<0,001)$, sin diferencias entre las fechas. Las especies nuevas registradas en los bosques de $P$. parlatorei en 2007 fueron Azara salicifolia, Prunus tucumanensis y Solanum sp. y en los bosques de A. acuminata - C. tucumanum fueron $P$. tucumanensis y $P$. persica.

Cuadro 3. Coeficientes de correlación Tau de Kendall entre la abundancia de especies de dosel por bosque y los valores de los bosques sobre los ejes del ordenamiento de escalamiento multidimensional no métrico $(* P<0,05$, $* * P<0,01)$.

Kendall Tau coefficient correlations between canopy species abundance by forest and forest axis scores values in non metric multidimensional scaling ordination $\left(* P<0.05\right.$, $\left.{ }^{* *} P<0.01\right)$.

\begin{tabular}{lcc}
\hline \multicolumn{1}{c}{ Especies } & Eje 1 & Eje 2 \\
\hline Alnus acuminata & $-0,65^{* *}$ & 0,02 \\
Blepharocalyx salicifolius & $0,56^{* *}$ & $-0,30^{* *}$ \\
Cedrela lilloi & $0,44^{* *}$ & 0,08 \\
Crinodendron tucumanum & $-0,36^{* *}$ & $-0,12$ \\
Dunalia lorentzii & 0,16 & $-0,55^{* *}$ \\
Ilex argentina & $0,37^{* *}$ & $-0,38^{* *}$ \\
Juglans Australis & $0,35^{* *}$ & 0,16 \\
Myrcianthes callicoma & $0,38^{* *}$ & $0,24^{*}$ \\
Myrcianthes mato & $0,51^{* *}$ & $-0,36^{* *}$ \\
Myrcianthes pseudo-mato & $0,38^{* *}$ & $-0,39^{* *}$ \\
Podocarpus parlatorei & $0,40^{* *}$ & $0,69^{* *}$ \\
Prunus tucumanensis & $0,29^{* *}$ & $-0,55^{* *}$ \\
Sambucus nigra & 0,02 & $-0,25^{*}$ \\
\hline
\end{tabular}




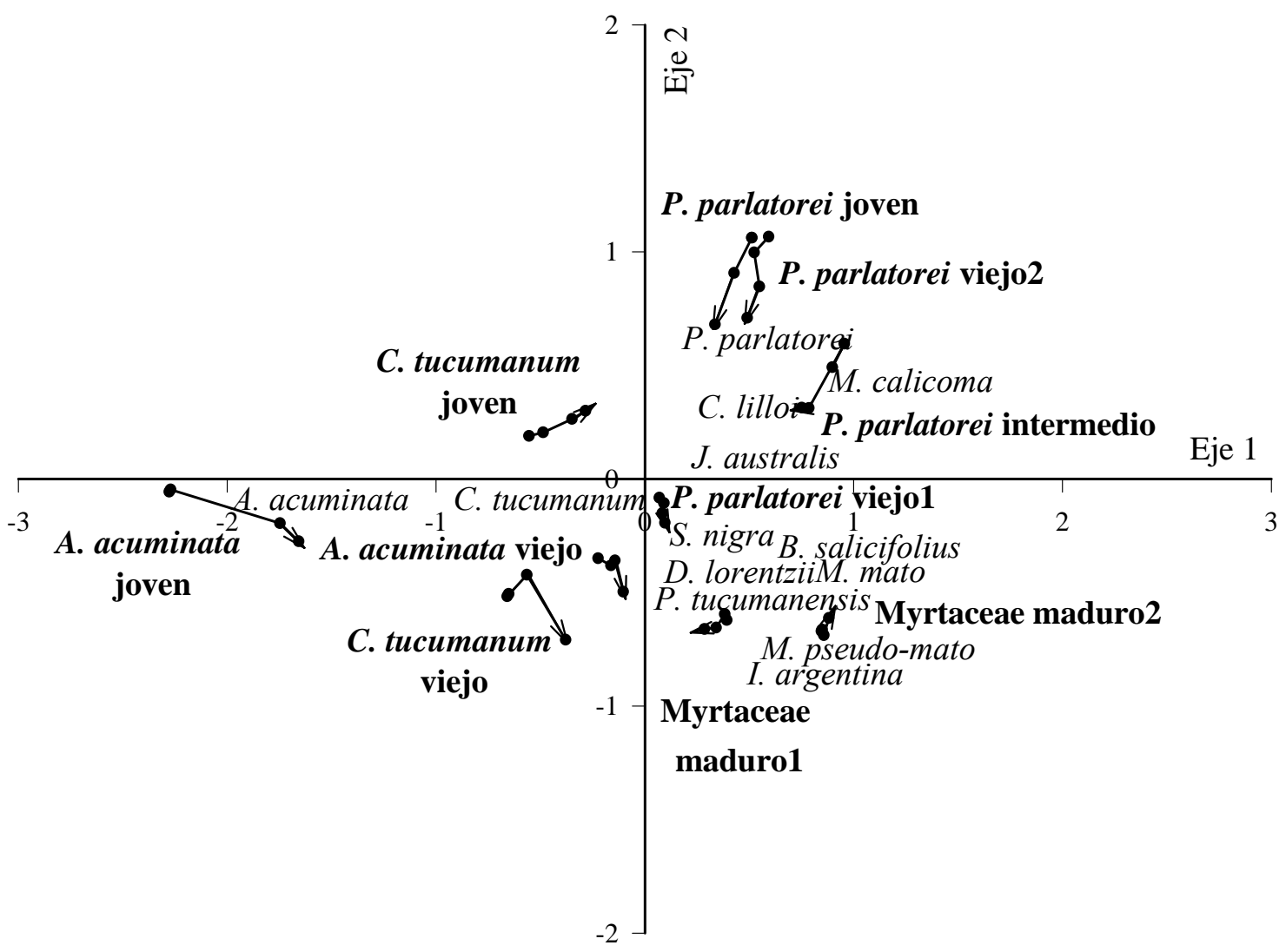

Figura 2. Diagrama de escalamiento multidimensional no métrico basado en la matriz de 13 especies de dosel en 10 parcelas por cuatro fechas 1991, 1996, 2001 y 2007. La escala de los ejes es proporcional a su contribución de la varianza explicada; el eje 1 explicó el $47 \%$ de la varianza y el eje 2 el $32 \%$. Cada flecha representa el cambio en la composición de cada bosque, en las distintas fechas. La posición de las especies en el diagrama se basa en su abundancia promedio, que se ajustó a una curva de abundancia unimodal a lo largo de cada eje. Los tipos de bosques están identificados en negrita.

Non-metrical multidimensional scaling (NMDS) diagram, biplot based on a matrix of 13 forest species composition in 1991, 1996, 2001 and 2007. Axis scale is proportional to their contribution in explaining variance; axis 1 explains $47 \%$ of the variance and axis 2 explains $32 \%$. Each arrow represents compositional changes (ordination diagram location) of each forest in different dates. Diagram species position is based on mean abundance that fits to a unimodal abundance curve along each axis. Forest types are identified by bold letter.

Tasas de mortalidad y reclutamiento. Los bosques viejos de A. acuminata y C. tucumanum presentaron elevadas tasas anuales de mortalidad (3,2\%) y reclutamiento (6,5 \%). El bosque viejo de $A$. acuminata registró el mayor número de reclutamientos (454 árboles/ha en 16 años), dado principalmente por la especie de sotobosque Solanum grossum (87 \% del total reclutado). El bosque joven de C. tucumanum presentó ambas tasas con valores intermedios (2,8 y $4,9 \%$, respectivamente), mientras que en el bosque joven de $A$. acuminata ambas tasas fueron relativamente bajas y similares entre sí (1,7 y 1,4 \%). El bosque de P. parlatorei joven y el de etapa sucesional intermedia presentaron ambas tasas similares (2,3 y 2,0 \% en promedio, respectivamente). Los bosques viejos de P. parlatorei presentaron una tasa de mortalidad intermedia ( $2,4 \%$ en promedio), pero la tasa más elevada de reclutamiento (promedio 3,3 \%). Los bosques maduros de mirtáceas registraron ambas tasas in- termedias y equilibradas entre sí (cuadro 1). Resumiendo, las tasas de mortalidad fueron similares entre los distintos tipos de bosques (de 2,3 a 2,7 \%), mientras que, las tasa de reclutamiento presentaron un rango de valores más amplio, de 2,1 \% (bosques de mirtáceas) a 4,8 \% (bosques dominados por A. acuminata y C. tucumanum). Las tasas de recambio siguieron este patrón, siendo mayores en los bosques viejos de $A$. acuminata y C. tucumanum (4,9 \%), y menores en los demás bosques (de 2 a $2,7 \%$; cuadro 1 ).

Área basal y biomasa. Los bosques de A. acuminata y C. tucumanum presentaron los valores más bajos de área basal (entre 20,2 y 26,3 m²/ha en 2007) y también los cambios más marcados entre 1991 y 2007, aumentando en los bosques jóvenes (5,5 y 3,9 $\mathrm{m}^{2} /$ ha, respectivamente) y disminuyendo en los bosques viejos $\left(-3,9\right.$ y $-1,8 \mathrm{~m}^{2} / \mathrm{ha}$, respectivamente). Los bosques de $P$. parlatorei registraron 


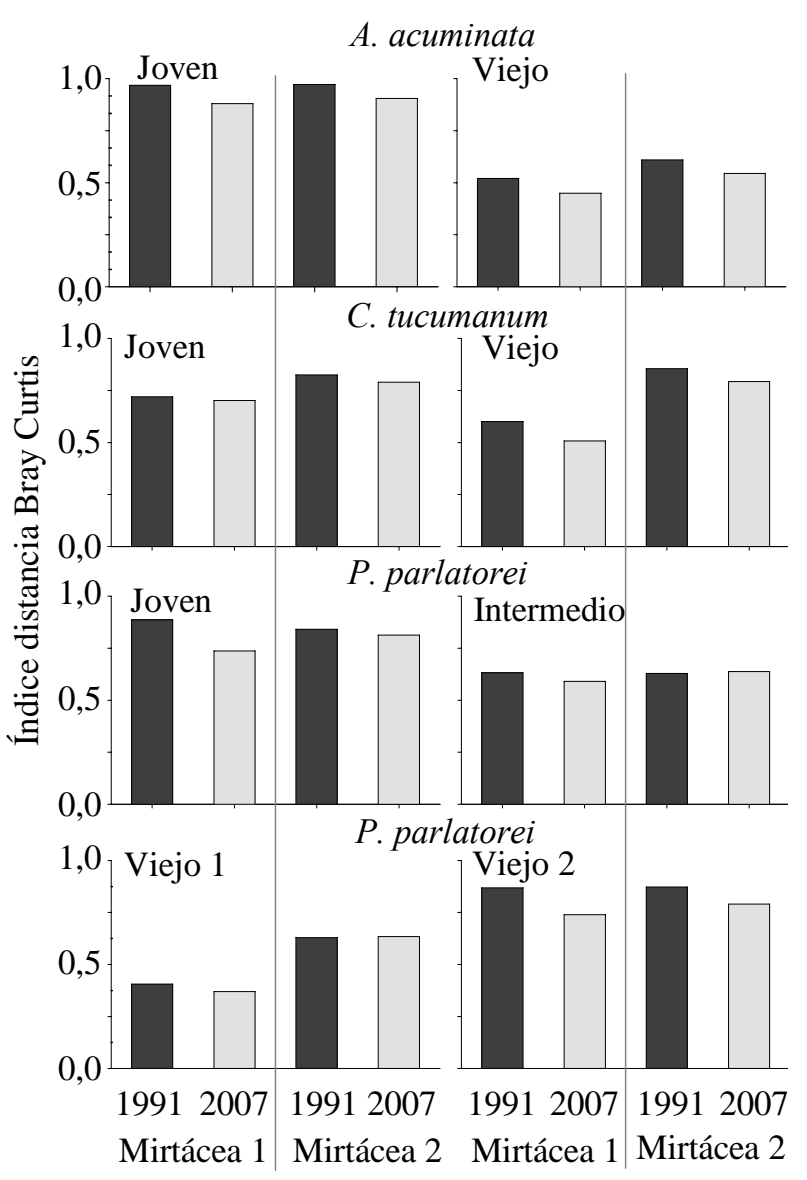

Figura 3. Índice de distancia Bray Curtis calculado entre bosques secundarios y maduros de mirtáceas 1 y 2 en 1991 y 2007, considerando las especies del dosel.

Bray Curtis index calculated between secondary forest and old growth Myrtaceae forest in 1991 and 2007, only considering canopy species.

los valores más elevados de $\mathrm{AB}$ (promedio: 43,9 m²/ha), con cambios marcados en el bosque joven $\left(5,1 \mathrm{~m}^{2} / \mathrm{ha}\right)$ y cambios menores en los bosques viejos. Los bosques de mirtáceas con un valor de $\mathrm{AB}$ intermedia respecto de los otros bosques (promedio: $36,2 \mathrm{~m}^{2} / \mathrm{ha}$ ), presentaron cambios menores en $\mathrm{AB}$ en el periodo evaluado (cuadro 1).

La biomasa aérea estimada difirió entre los bosques y entre las especies y siguió patrones similares al área basal en la que está basada su estimación. El bosque de P. parlatorei en etapa sucesional intermedia registró el valor más elevado en 2007 (623,2 t/ha), y el bosque joven de A. acuminata el más bajo (137,5 t/ha). Los mayores incrementos de biomasa en 16 años se registraron en los bosques jóvenes de $P$. parlatorei (47,9 t/ha) y C. tucumanum $(47,1$ $\mathrm{t} / \mathrm{ha})$, en el bosque de $P$. parlatorei intermedio (45,5 t/ha) y de mirtáceas (46,6 t/ha). En este último, el aumento estuvo influido por pocos individuos de grandes dimensiones. Los mayores aumentos en la biomasa relativa en relación a la de 1991 se registraron en los bosques jóvenes de $A$. acuminata (44,6 \%), C. tucumanum (30,2 \%) y P. parlatorei
(19,5 \%), como se esperaría para bosques jóvenes más dinámicos, y las mayores pérdidas en los bosques viejos de A. acuminata (-21,9 \%) y C. tucumanum (-13,6 \%). Los bosques viejos de $P$. parlatorei mantuvieron relativamente constante su biomasa en el tiempo (0,2 y 9,4 t/ha, entre 2007 y 1991; cuadro 1). Las especies que más biomasa aportaron a los bosques promediando todas las fechas fueron P. parlatorei (160,7 t/ha), C. tucumanum (75,8 t/ ha), M. mato (52 t/ha) y A. acuminata $(48,5 \mathrm{t} / \mathrm{ha}$; figura 4A). Entre 1991 y 2007, 11 especies disminuyeron su biomasa, principalmente, C. tucumanum (-11 t/ha), A. acuminata (-10,5 t/ha), P. parlatorei (-7 t/ha), M. mato (-6,3 t/ha) y $D$. lorentzii $(-5,2 \mathrm{t} / \mathrm{ha})$. Las especies que aumentaron su biomasa fueron principalmente $S$. grossum $(6,7 \mathrm{t} / \mathrm{ha})$ que duplicó su valor en este periodo (119\%), M. pseudomato (7,4 t/ha) y M. calicoma (4,26 t/ha; figura 4B).

Demografía, estructura de tamaños y crecimiento de Podocarpus parlatorei. Esta especie presentó tasas demográficas relativamente bajas, con variaciones según el tipo de bosque. La tasa de mortalidad promedio de $P$. parlatorei fue de $1,8 \%$ y de reclutamiento de $0,5 \%$, para el periodo 1991 - 2007. El bosque joven de esta especie presentó las tasas de mortalidad (1,9\%) y de reclutamiento (0,5 \%) más elevadas y los bosques viejos registraron la tasa de reclutamiento más baja $(0,1 \%)$, aún más que en los de bosques de mirtáceas (0,8 \%). En los bosques de C. tucumanum - A. acuminata, las tasas de mortalidad de $P$. parlatorei fueron bajas $(0,1 \%)$. Sin embargo, en los bosques viejos correspondientes, se registró la tasa de reclutamiento más alta (3,1\%), por el reclutamiento de pocos individuos de esta especie en el último periodo en el bosque viejo de $C$. tucumanum, donde no había individuos de P. parlatorei antes de 2007.

La distribución de tamaños de $P$. parlatorei (incluido individuos multifustales con DAP virtual), varió según la edad de los bosques y en menor medida entre ambas fechas (1991 y 2007). En 1991, el bosque joven de P. parlatorei presentó una distribución de tipo j invertida, con mayor proporción de individuos pequeños, que reemplazarían a los individuos grandes en el futuro. Sin embargo, en 2007 la distribución de tamaños se revirtió, disminuyendo la proporción de individuos jóvenes, como muestran los demás bosques. En términos generales, se observó un envejecimiento de la población de P. parlatorei, con pocos individuos reclutando y dominando árboles grandes, como ocurre en los bosques viejos, donde la distribución fue unimodal, con mayor porcentaje de individuos de diámetros medios (figura 5).

La tasa de crecimiento anual calculada en base al DAP virtual para $P$. parlatorei fue relativamente baja $(0,2 \pm$ $0,2 \mathrm{~cm}$ ) en los primeros 10 años, con un leve aumento en los últimos seis años $(0,3 \pm 0,2 \mathrm{~cm})$. Solanum grossum $y$ $P$. tucumanensis presentaron las tasas más elevadas $(0,4$ $\pm 0,3 \mathrm{~cm})$, seguidas por $A$. acuminata $(0,3 \pm 0,3 \mathrm{~cm})$. Las especies que menos crecieron fueron $M$. mato $(0,12 \pm 0,19$ cm) y B. salicifolia $(0,13 \pm 0,28 \mathrm{~cm})$. 


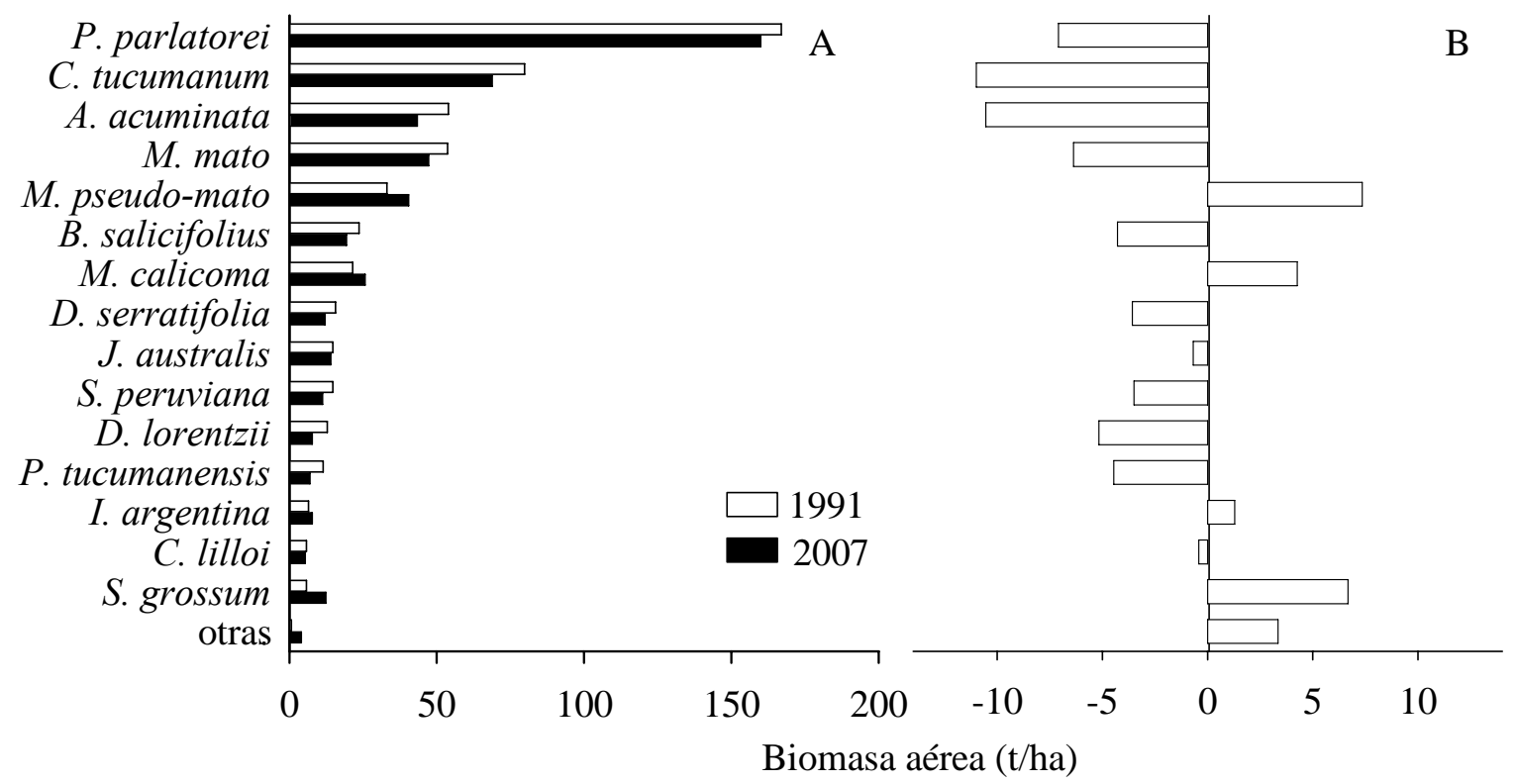

Figura 4. Biomasa aérea de las 15 especies más abundantes, A) en 1991 y 2007 y B) cambio en biomasa entre 1991 y 2007. El término otras, en el eje x está referido a las siete especies restantes cuya biomasa aérea es $\leq 1 \mathrm{t} /$ ha (apéndice 1).

Aboveground biomass of the most abundant 15 species, A) in 1991 and 2007, and B) biomass change between 1991 and 2007. "Otras" (others), in axis $\mathrm{x}$ is referred to seven species with biomass $\leq 1 \mathrm{t} / \mathrm{ha}$ (appendix 1 ).

\section{DISCUSIÓN}

El ordenamiento de los bosques montanos de la sierra de San Javier estudiados, de acuerdo a los cambios temporales de su composición, sugiere la existencia de dos tendencias sucesionales con una convergencia hacia los bosques maduros de mirtáceas. Por un lado, estarían los bosques dominados por $P$. parlatorei y, por otro, los bosques dominados por A. acuminata y C. tucumanum, comparativamente similares en cuanto a su dinámica sucesional. En este último grupo, el bosque jóven de A. acuminata es el más diferente, posiblemente por la abundancia y pérdida de esta especie (de $96 \%$ en 1991 a $88 \%$ individuos en 2007), acompañada del aumento de Sambucus nigra (con tasas de reclutamiento de $8 \%$ ). Los bosques maduros de mirtáceas fueron los más estables reflejado en sus cortas trayectorias sucesionales, tal como se esperaría para bosques maduros (Oliver y Larson 1996).

Siguiendo el modelo de Oliver y Larson (1996), Grau (2006) clasificó al patrón sucesional de los bosques dominados por $P$. parlatorei en cuatro etapas; i) fase de iniciación de rodal, donde el área abandonada (principalmente ganadera) es colonizada por arbustos y ocasionalmente por A. acuminata, facilitando el establecimiento inicial de P. parlatorei; ii) fase de autorraleo, caracterizada por una intensa competencia intraespecífica, y escaso reclutamiento de individuos arbóreos bajo un dosel cerrado; iii) fase de reiniciación, derivada de la mortalidad de especies del dosel por competencia que permite el establecimiento de especies en el sotobosque; y iv) fase de bosque maduro, donde los claros producidos por la caída de árboles viejos resulta en el establecimiento de especies tolerantes o semitolerantes a la sombra. Siguiendo este modelo, los bosques de $P$. parlatorei observados en este estudio se encontrarían en la fase de autorraleo, en transición con fase de reiniciación, reflejada en la dinámica del sotobosque.

Aunque con tasas variables de cambio, se observa una tendencia hacia una convergencia composicional, reflejada en los índices de similitud entre bosques secundarios y de mirtáceas. Esta tendencia sucesional ya fue descrita por otros autores quienes describen a los bosques dominados por A. acuminata como los más simples, a los dominados por mirtáceas, más complejos y diversos, y en una posición intermedia del gradiente sucesional, los bosques de P. parlatorei y C. tucumanum (Arturi et al. 1998). En este estudio, $C$. tucumanum se asemeja más a un bosque simple de A. acuminata que a uno de P. parlatorei.

Los valores bajos de área basal en los bosques jóvenes de A. acuminata y C. tucumanum son similares a los de otros bosques montanos secundarios dominados por estas especies (26,9 m²/ha, Morales y Brown 1998). Las relativamente altas tasas de acumulación de biomasa registradas para estos bosques son características de bosques en estadio sucesional temprano. El bosque joven de P. parlatorei se encuentra en una etapa sucesional más avanzada (> 200 años) y se comporta de manera similar a los bosques jóvenes mencionados en cuanto a la acumulación de biomasa, pero presenta valores de área basal más elevados. En el bosque joven de A. acuminata el incremento de área basal se debería al crecimiento diametral de Sambucus nigra 

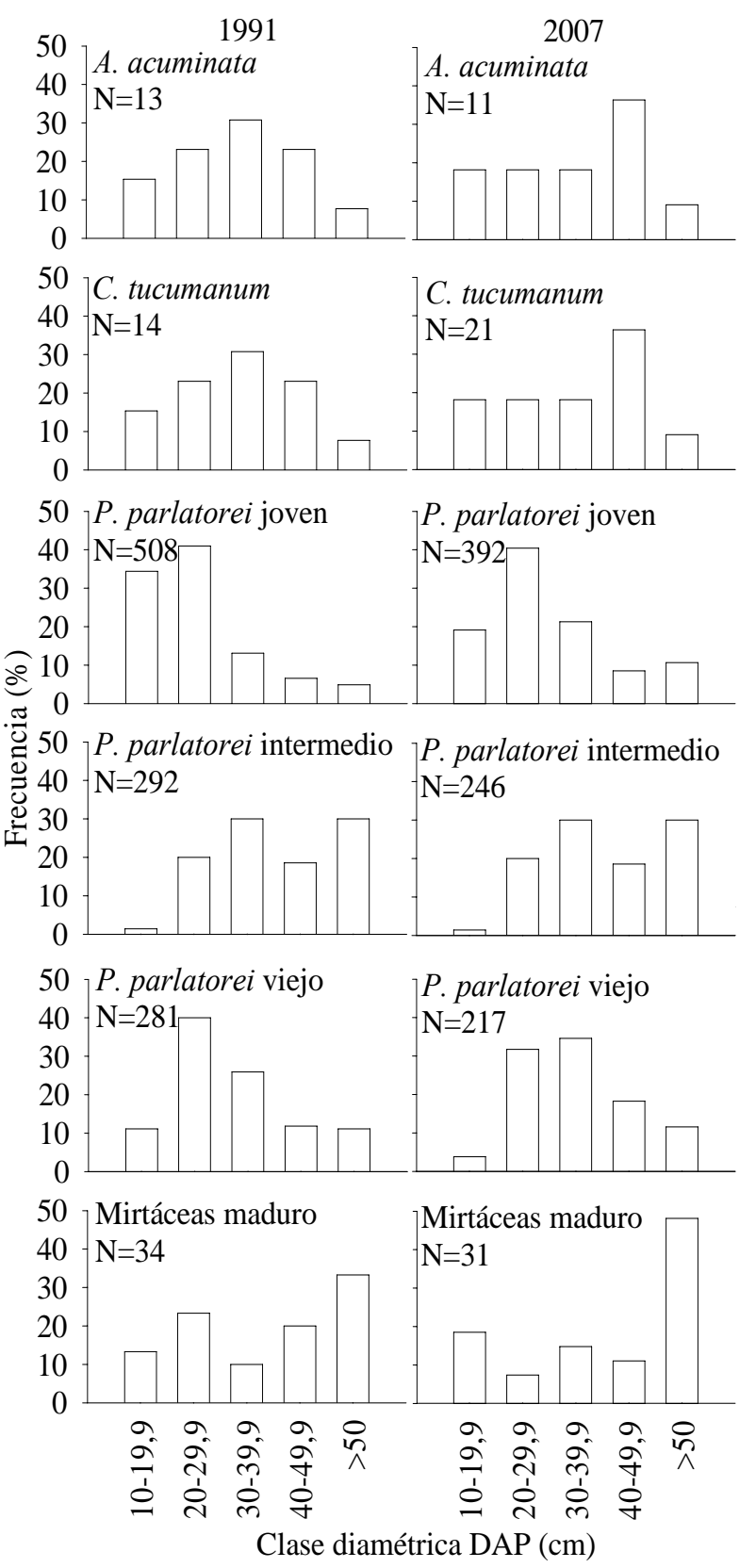

Figura 5. Distribución de clases diametrales de $P$. parlatorei en 1991 y 2007. La distribución de diámetros se expresa como frecuencia relativa de las clases diametrales cada $10 \mathrm{~cm}$. Los porcentajes se calcularon a base del número total de individuos de $P$. parlatorei por bosque $(\mathrm{N})$. Bosques jóvenes y viejos de A. acuminata y C. tucumanum se graficaron juntos por la poca abundancia de individuos de $P$. parlatorei presentes.

Distribution of P. parlatorei diametric classes in 1991 and 2007. Diametric distribution is expressed as relative frequency of $10 \mathrm{~cm}$ diametric classes. Percentages were calculated considering $P$. parlatorei individuals $(\mathrm{N})$. Young and old A. acuminate and C. tucumanum forests were graphed together because of the few individuals of $P$. parlatorei present.
(0,4 cm/año, todos los individuos de 12 a $21 \mathrm{~cm}$ DAP). Por el contrario, en el bosque joven de $P$. parlatorei el aumento en área basal se debe principalmente al elevado reclutamiento de nuevos individuos (3,1\%; cuadro 1). En el bosque joven de C. tucumanum el aumento de área basal se explica por la combinación de ambos factores: elevada tasa de reclutamiento $(6,5 \%)$ y de crecimiento diametral de dos especies abundantes (P. parlatorei y S. grossum con 0,7 y $0,3 \mathrm{~cm} / a n ̃ o$, respectivamente). En este bosque, la tasa de crecimiento de $P$. parlatorei fue más elevada que el promedio registrado para esta especie en los demás bosques (0,3 cm anuales), y a valores registrados para otra especie de Podocarpus (e.g. P. nubigenus 0,2 a 0,3 cm/año) en bosques de Chile (Enright y Orden 1995).

En contraste, los bosques viejos de A. acuminata y $C$. tucumanum registraron una reducción en área basal y en biomasa. Estos bosques acumulan biomasa rápidamente en etapas tempranas de sucesión, reflejado en el elevado porcentaje de biomasa en los bosques jóvenes. En parte, esta biomasa no se conserva debido por un lado, a la corta longevidad de las especies dominantes (principalmente $A$. acuminata) y por el otro, a que los individuos no son reemplazados por otras especies arbóreas de dosel, reflejado en la pérdida de biomasa de los bosques en etapa sucesional más avanzada, sugiriendo un quiebre estructural, donde dominarían especies de estratos arbóreos más bajos. Estos resultados son consistentes con otros bosques montanos de Argentina, donde la recuperación composicional podría tardar más tiempo que la recuperación estructural (Grau et al. 1997, Easdale 1999, Grau 2006), principalmente por la baja tasa de reclutamiento de especies de bosques maduros, que podría estar retardando la sucesión hacia la composición de estos bosques. En este sentido, se destaca la gran expansión poblacional de Solanum grossum, con elevado reclutamiento en todos los bosques (86 \%), es una especie de vida corta que raramente supera los $30 \mathrm{~cm}$ DAP. Consecuentemente, S. grossum, no llegaría a reemplazar a especies longevas de dosel; aportaría poco en la regeneración del bosque (Chazdon et al. 2010) y en nuestro estudio no contribuyó a explicar las tendencias florísticas, pues estas están basadas en especies del dosel. En menor medida, Sambucus nigra y Dunalia lorentzii, que reclutan abundantemente pero no alcanzan gran tamaño reflejan este mismo patrón de un cambio en la estructura dominado por el aumento significativo de arbolitos del sotobosque. Estas especies sugieren la importancia relativa del sotobosque en relación a las del dosel, sin embargo, su efecto en la dinámica sucesional no es claro; podrían tener un efecto competitivo con las especies del dosel en estadios tempranos de vida, limitando la velocidad de regeneración de la composición del bosque maduro o un efecto inverso, favoreciendo la llegada de ciertas especies, al promover la recuperación del suelo, el ciclado de nutrientes y la comunidad de dispersores (Clark et al. 2004).

En contraposición, los bosques viejos de P. parlatorei con elevados valores de biomasa y área basal, estables en 
Cuadro 4. Número de individuos reclutados y muertos en 16 años para los distintos tipos de bosques. Number of recruited and dead individuals in 16 years for each forest type.

\begin{tabular}{|c|c|c|c|c|c|c|}
\hline \multirow[t]{2}{*}{ Especies } & $\begin{array}{l}\text { A. acuminata, } \\
\text { C. tucumanum }\end{array}$ & P. parlatorei & Mirtáceas & $\begin{array}{l}\text { A. acuminata, } \\
\text { C. tucumanum }\end{array}$ & P. parlatorei & Mirtáceas \\
\hline & \multicolumn{3}{|c|}{ Reclutados } & \multicolumn{3}{|c|}{ Muertos } \\
\hline A. acuminata & 12 & 0 & 0 & 38 & 1 & 0 \\
\hline B. salicifolius & 0 & 0 & 1 & 0 & 1 & 5 \\
\hline C. lilloi & 0 & 3 & 0 & 0 & 0 & 0 \\
\hline C. tucumanum & 0 & 9 & 0 & 4 & 1 & 3 \\
\hline D. lorentzii & 12 & 4 & 11 & 6 & 3 & 16 \\
\hline D. serratifolia & 0 & 0 & 2 & 0 & 0 & 10 \\
\hline K. lasiophthalma & 4 & 0 & 0 & 2 & 0 & 0 \\
\hline M. mato & 0 & 0 & 8 & 0 & 1 & 26 \\
\hline P. parlatorei & 7 & 4 & 3 & 2 & 59 & 7 \\
\hline P. tucumanensis & 4 & 1 & 1 & 0 & 0 & 2 \\
\hline S. grossum & 212 & 89 & 83 & 47 & 28 & 33 \\
\hline S. nigra & 15 & 38 & 9 & 11 & 8 & 12 \\
\hline Otras & 1 & 6 & 1 & 0 & 1 & 2 \\
\hline Total & 267 & 154 & 119 & 110 & 103 & 116 \\
\hline
\end{tabular}

Bosques de A. acuminata - C. tucumanum (0,96 ha), de P. parlatorei (0,84 ha) y de mirtáceas (0,88 ha). Se muestran las 12 especies con más de un individuo reclutado y/o muerto en al menos uno de los bosques. "Otras" se refiere a las siete especies restantes que solo presentaron un individuo muerto o reclutado. El total está calculado para las 19 especies presentes en los bosques.

Alnus acuminata - C. tucumanum forest (0.96 ha), P. parlatorei forest ( $0.84 \mathrm{ha})$ and Mirtaceae forest ( $0.88 \mathrm{ha})$. The 12 species with more than one recruited and /or dead individuals are indicated. "Otras" (others) is referred to the seven species that presented only one recruited or dead individual. Total is calculated for the 19 species presented in forest.

el tiempo, tienen capacidad para secuestrar biomasa lentamente y consistente a largo plazo. De la misma manera, el área basal elevada muestra pequeños cambios temporales, sugiriendo que estos bosques estuvieron experimentando una intensa competencia intraespecífica (fase de autorraleo), que resulta en una tasa de sucesión lenta y con lento crecimiento de individuos dominantes, tendiendo a la predominancia de individuos viejos. Eventualmente, comenzarían a producirse claros por mortalidad de árboles grandes, liberando recursos y brindando oportunidades para nuevos individuos y mayor crecimiento de árboles (fase de reiniciación) como se observa en bosques templados (Oliver y Larson 1996, Gutierrez et al. 2008). Este patrón ha sido descrito por Grau (2006) donde las prolongadas fases de autorraleo y de reiniciación de sotobosque dominarían la mayoría de los bosques de $P$. parlatorei de la región. Estos bosques, que se encuentran en estadios sucesionales más avanzados, alcanzaron elevados valores de área basal (44 m²/ha), semejantes, e incluso mayores, a los bosques maduros de mirtáceas ( $37 \mathrm{~m}^{2} / \mathrm{ha}$ ), comparables con bosques de $P$. parlatorei maduros de la alta cuenca del río Bermejo (41,4 m²/ha; Pinazo et al. 2003) y superiores a bosques secundarios de esta región ( $27 \mathrm{~m}^{2} / \mathrm{ha}$; Pinazo et al. 2003 y 26,9 m²/ha; Morales y Brown 1998). Los bosques dominados por $P$. parlatorei de la sierra de San Javier, presentan los valores máximos de área basal registrados para los bosques subtropicales del NO argentino hasta la fecha.

Los cambios temporales de biomasa registrados en los bosques más jóvenes, indicarían que funcionan fijando carbono. Mientras que, los bosques viejos de $P$. parlatorei alcanzarían un equilibrio funcional manteniendo las fuen- tes de carbono acumuladas. Particularmente, el género Podocarpus presenta elevados porcentajes de carbono en sus tejidos; por ejemplo, Podocarpus nubigena Lindl. en bosques de Chile (Gayoso y Guerra 2005). El papel de los bosques maduros de mirtáceas en el balance de carbono no es tan claro; a pesar de ser relativamente estables, presentan tendencias variables. Algunos estudios proponen que los bosques maduros podrían funcionar como reservorios de carbono, siendo más vulnerables a disturbios y a factores climáticos (Phillips et al. 1998), teniendo un efecto sobre la mortalidad de árboles y consecuentemente sobre la biomasa del bosque (van Mantgem y Stephenson 2007). Wardle et al. (2004) proponen que en ausencia de disturbios por largos periodos, los bosques primarios y secundarios, entrarían en una fase de declinación de biomasa, acompañada con cambios en las cantidades relativas de nitrógeno y fósforo a lo largo de la sucesión. Un patrón similar se observa en Nueva Zelandia, donde los bosques aumentarían su biomasa a lo largo de la sucesión hasta alcanzar un máximo y después disminuirían a un nivel intermedio (Allen et al. 1997).

El dinamismo de los bosques de $P$. parlatorei está dado básicamente por las especies que más reclutan en el sotobosque: S. grossum y S. nigra, como en los otros bosques, aunque están siendo reemplazadas muy lentamente por especies de bosques maduros (por ejemplo, $C$. tucumanum, Cedrela lilloi y Prunus tucumanensis). Patrones similares se observaron en parches dominados por $P$. parlatorei de la alta cuenca del río Bermejo, con un lento reemplazo por especies del bosque maduro (Ramadori 1998). Un patrón interesante emerge de estos datos y debe ser tenido en cuenta para los próximos estudios, y 
es que una parte importante de la dinámica del bosque no ocurre en las especies del dosel, sino en las del sotobosque. Clasificar las especies en grupos funcionales puede contestar este tipo de preguntas y contribuir a entender los procesos de ensamblaje de la comunidad (Chazdon et al. 2010).

El clima y la dinámica de disturbios influyen en el establecimiento de las especies arbóreas. El establecimiento de A. acuminata es favorecido por el fuego, al disminuir la competencia con los pastos, y por las precipitaciones, que además de promover la expansión del bosque, favorece los incendios, al producir mayor biomasa vegetal combustible (Grau y Veblen 2000, Aráoz y Grau 2010). En cambio, P. parlatorei, tiende a estar favorecido por niveles relativamente altos de pastoreo y frecuencia baja de fuego (Carilla y Grau 2010), y una vez establecido persiste por varios siglos como especie dominante, alcanzando valores elevados de biomasa. Con el progreso de la sucesión, estos bosques tienden a envejecer y a ser reemplazados por bosques más diversos, reflejado en la distribución de tamaños, similar a bosques templados de Chile (Gutiérrez et al. 2008). Este comportamiento concuerda con otras coníferas del hemisferio sur, que en ausencia de grandes disturbios pueden declinar su población, dada su habilidad limitada para colonizar sitios con disturbios de pequeña escala y regenerar en el sotobosque (Enright y Orden 1995).

\section{CONCLUSIONES}

Las dos trayectorias sucesionales observadas difieren estructuralmente; una (dominada por Alnus acuminata y Crinodendron tucumanum) representa bosques con poca biomasa que aumenta rápidamente, pero no se conserva, porque la apertura del dosel dada por la mortalidad de árboles viejos es capitalizada por especies del sotobosque con limitada acumulación de biomasa, estabilizándose en una dinámica de alto reclutamiento y mortalidad. La otra (bosques dominados por Podocarpus parlatorei) refleja bosques secundarios en estadios sucesionales más avanzados, que han acumulado gran cantidad de biomasa en la etapa de competencia intraespecifica (exclusión fustal) y se mantiene en una etapa de reiniciación del sotobosque, pese a la muerte de árboles. Con el avance de la sucesión, la biomasa tiende a disminuir al morir grandes individuos, ofreciendo oportunidades para que se recupere el bosque maduro pero a tasas muy lentas. La composición de los bosques sucesionales tiende a converger hacia la de bosques maduros, reemplazando muy lentamente especies de sotobosque por típicas de dosel. Los resultados enfatizan la importancia de estudios a largo plazo para entender la dinámica de los bosques de montaña, y el manejo por sus servicios ecosistémicos. Principalmente la importancia de los bosques en el balance de carbono a lo largo del gradiente sucesional, sus tasas de recuperación para la conservación de la biodiversidad y protección de cuencas.

\section{AGRADECIMIENTOS}

Este estudio fue financiado por el Consejo Nacional de Investigaciones Científicas y Técnicas (CONICET) y la Agencia Nacional de Promoción Científica y Tecnológica (FONCYT). Sofía Olea, Ezequiel Aráoz, Sofia Marinaro, Gustavo Sánchez, Carolina Jamroz y estudiantes de la Facultad de Agronomía y Zootecnia, UNT, colaboraron con el trabajo de campo.

Cuatro revisores anónimos aportaron valiosos comentarios al manuscrito.

\section{REFERENCIAS}

Aide TM, HR Grau. 2004. Globalization, migration and Latin American ecosystems. Science 305: 1915-1916.

Allen RB, PW Clinton, MR Davis. 1997. Cation storage and availability along a Nothofagus forest development sequence in New Zealand. Canadian Journal of Forest Research 27: 323-330.

Aráoz E, HR Grau. 2010. Fire-mediated forest encroachment in response to climate and land-use change in subtropical Andean Treelines. Ecosystems 13: 992 - 1005.

Arturi MF, HR Grau, PG Aceñolaza, AD Brown. 1998. Estructura y sucesión en bosques montanos del Noroeste de Argentina. Revista de Biología Tropical 46(3): 525-532.

Bellingham PJ, AD Sparrow. 2009. Multi-stemmed trees in montane rain forests: their frequency and demography in relation to elevation, soil nutrients and disturbance. Journal of Ecology 97: 472-483.

Brown AD, HR Grau, LR Malizia, A Grau. 2001. Argentina. In Kappell M, $\mathrm{AD}$ Brown eds. Bosques Nublados del Neotrópico. San José, Costa Rica. INBIO. p. 622 - 659

Cabrera, AL. 1976. Regiones fitogeográficas argentinas. Buenos Aires, Argentina. ACME. 85 p.

Cabrera AL, A Willink. 1980. Biogeografía de América Latina. Washington DC, USA. Organización de los Estados Americanos. 122 p.

Carilla J, HR Grau, A Malizia. 2006. Successional rates in postgrazing secondary forests in NW Argentina mountains. In Sphen E, M Liberman, C Körner eds. Land use change and mountain biodiversity. Floria, USA. Taylor and Francis Group. p. $261-271$.

Carilla J, HR Grau. 2010. 150 years of tree establishment, land-use and climate changes in montane grasslands, NW Argentina. Biotropica 42(1): 49-58.

Chave J, HC Muller-Landau, TR Baker, TA Easdale, H ter Steege, CO Webb. 2005. Regional and phylogenetic variation on wood density across 2456 neotropical tree species. Ecological Applications 16(6): 2356-2367.

Chazdon RL. 2003. Tropical forest recovery: legacies of human impact and natural disturbances. Perspectives in Plant Ecology, Evolution and Systematics 6(1-2): 51-71.

Chazdon RL, B Finegan, RS Capers, B Salgado-Negret, F Casanoves, V Boukili1, N Norden. 2010. Composition and dynamics of functional groups of trees during tropical forest succession in Northeastern Costa Rica. Biotropica 42 (1): $31-40$

Clark CJ, JR Poulsen, EF Connor, VT Parker. 2004. Fruiting 
trees as dispersal foci in a semi-deciduous tropical forest. Oecologia 139: 66-75.

Condit R. 1995. Research in large, long-term tropical forest plots. Tree 10 (1):10-22

Condit R, SP Hubbell, RB Foster.1995. Demography and harvest potential of Latin American timber species: data from a large permanent plot in Panama. Journal of Tropical Forest Science 7(4): 599-622

Crk T, M Uriarte, F Corsi, D Flynn. 2009. Forest recovery in a tropical landscape: what is the relative importance of biophysical, socioeconomic, and landscape variables? Landscape Ecology 24: 629-642.

Easdale TA. 1999. Relación de disturbios y factores ambientales con la diversidad, composición y estructura de comunidades leñosas en el Valle de Los Toldos, Yungas Argentinas. Tesis de Licenciatura. Tucumán, Argentina. Laboratorio de Investigaciones Ecológicas de las Yungas, Universidad Nacional de Tucumán. 21 p.

Easdale TA, DE Gurvich, AN Sersic, JR John. 2007. Tree morphology in seasonally dry montane forest in Argentina: Relationships with shade tolerance and nutrient shortage Journal of Vegetable Science 18: 313-326.

Enright NJ, J Ogden. 1995. The Southern conifers - A synthesis. In NJ Enright and RS Hill eds. Ecology of the southern conifers. Melbourne, Australia. Melbourne University Press. p. 271- 287.

Finegan B, D Delgado. 2000. Structural and floristic heterogeneity in a 30-year-old Costa Rican rain forest restored on pasture through natural secondary sucession. Restoration Ecology 8(4): 380-393.

Gayoso JA, J Guerra. 2005. Contenido de carbono en la biomasa aérea de bosques nativos en Chile. Bosque 26(2): 33-38

Giusti L, A Slanis, PG Aceñolaza. 1997. Fitosociología de los bosques de aliso (Alnus acuminata H.B.K. ssp. acuminata) de Tucumán, Argentina. Lilloa 38: 93-120.

Grau A. 1985. La expansión del aliso del cerro (Alnus acuminata H. B. K. subsp. acuminata) en el Noroeste de Argentina. Lilloa 36: 237-247.

Grau HR, L Paolini, A Malizia, J Carilla. 2010. Distribución, estructura y dinámica de los bosques de la Sierra de San Javier. In Grau HR ed. Ecología de una transición naturalurbana: El Gran San Miguel de Tucumán y la Sierra de San Javier. San Miguel de Tucumán, Argentina. Editorial de la Universidad Nacional de Tucumán. p. 33-48.

Grau HR, MF Arturi, AD Brown, PG Aceñolaza. 1997. Floristic and structural patterns along a chronosequence of secondary forest succession in Argentinean subtropical montane forests. Forest Ecology and Management 95(2): 161-171.

Grau HR, T Veblen. 2000. Rainfall variability, fire and vegetation dynamics in neotropical montane ecosystems in northwestern Argentina. Journal of Biogeography 27: 11071121.

Grau HR, TM Aide, JK Zimmerman, JR Thomlinson. 2004. Trends and scenarios of the carbon budget in postagricultural Puerto Rico (1936-2060). Global Change Biology 10:1163-1179.

Grau HR. 2006. Dinámica de Bosques en el gradiente altitudinal de las Yungas Argentinas. In Arturi MF, JL Frangi, JF Goya eds. Ecología y manejo de los Bosques de Argentina. Buenos Aires, Argentina. p. 1-30.

Grau HR, NI Gasparri, MM Morales, A Grau, E Araoz, J Carilla y J Gutierrez. 2007. Regeneración ambiental en el Noroeste Argentino. Ciencia Hoy 17:42-55.

Grau HR, ME Hernández, J Gutierrez, NI Gasparri, MC Casavecchia, EE Flores, L Paolini. 2008. A peri-urban Neotropical forest transition and its consequences for environmental services. Ecology and Society 13(1): 35. URL: http://www.ecologyandsociety.org/vol13/iss1/art35/

Grau HR, L Paulini, A Malizia, J Carilla. 2010. Distribución, estructura y dinámica de los bosques de la Sierra de San Javier. In Grau HR ed. Ecología de una transición naturalurbana: El Gran San Miguel de Tucumán y la Sierra de San Javier. San Miguel de Tucumán, Argentina. Editorial de la Universidad Nacional de Tucumán. p. 38-48.

Grau HR, R Villalba, R Gil Montero, E Aráoz, J Carilla, M. Menbiela. 2010. Environmental history and forest regeneration in a degraded valley in the subtropical Argentinean cloud forest life zone. In Bruijnzeel LA, FN Scatena, LS Hamilton eds. Tropical montane cloud forest: Science for conservation and management. Cambridge, UK. Cambridge University Press. p. 597-604.

Guariguata MR, R Ostertag. 2001. Neotropical secondary forest succession: changes in structural and functional charasteristics. Forest Ecology and Management 148: 185206.

Gutierrez AG, JC Aravena, NV Carrasco-Farías, DA Christie, M Fuentes, JJ Armesto. 2008. Gap-phase dynamics and coexistence of a long-lived pioneer and shade-tolerant tree species in the canopy of an old-growth coastal temperate rain forest of Chiloé Island, Chile. Journal of Biogeography 35(9): 1674-1687.

Huzinger H. 1997. Hydrology of montane forests in the Sierra de San Javier, Tucuman, Argentina. Mountain research and development 17: 299-308.

Kruskal JB, M Wish. 1978. Multidimensional scaling. Sage University Papers series on Quantitative Applications in the Social Sciences, 07-011. Beverly Hills, USA. Sage Publications.

Legendre P, L Legendre. 1998. Numerical Ecology (2 ${ }^{\text {nd }}$ edition). Amsterdam, NL. Elsevier Science, 853 p.

Labraga JC. 1997. The Climate change in South America due to doubling in the $\mathrm{CO}_{2}$ concentration: intercomparison of general circulation model equilibrium experiments. International Journal of Climatology 17: 377-398.

McCune B, MJ Mefford. 1999. PC-ORD. Multivariate analysis of ecological data. MjM Software Design. Gleneden Beach, OR, USA. 237 p.

McCune B, Grace JB. 2002. Analysis of ecological communities. MjM Software Design, Gleneden Beach, OR, USA. 469 p.

Minetti JL, WM Vargas. 1997. Trends and jumps in the annual precipitation in South America, south of the $15^{\circ} \mathrm{S}$. Atmosfera 11(4): 205-221.

Morales JM, AD Brown. 1998. Bosques montanos con diferente intensidad de explotación. Bosques y Desarrollo 17: 51-52.

Moyano MY, CP Movia. 1989. Relevamiento fisonómico estructural de las sierras de San Javier y El Periquillo (Tucumán, Argentina) I: Área de las Yungas. Lilloa 37: 123-135.

Oliver CD, BC Larson. 1996. Forest stand dynamics. Update Edition. New York, USA. John Wiley. 540 p.

Pinazo MA, NJ Gasparri, JF Goya, MF Arturi. 2003. Caracterización estructural de un bosque de Podocarpus 
parlatorei y Juglans australis en Salta, Argentina. Revista de Biologia Tropical 51(2): 361-368.

Phillips O L, P Hall, AH Gentry, SA Sawyer, R Vasquez. 1994. Dynamics and species richness of tropical rain forests. PNAS 91(7): 2805-2809

Phillips OL, AH Gentry. 1994. Increasing turnover through time in tropical forest. Science 263: 954-958

Phillips OL,Y Malhi, N Higuchi, WF Laurance, PV Nuniez, RM Vasquez, SG Laurance, LV Ferreira, M Stern, S Brown, J Grace. 1998. Changes in the Carbon balance of tropical forests: evidence from long-term plots. Science 282: 439442.

Ramadori ED. 1998. Sucesión secundaria en bosques montanos del Noroeste Argentino. Tesis doctoral. La Plata Argentina. Facultad de Ciencias Naturales, Universidad Nacional de La Plata, Argentina. 474 p.

Sheil D, DFRP Burslem, D Alder. 1995. The interpretation and misinterpretation of mortality rate measures. Journal of Ecology 83: 331-333.

Silver WL, R Ostertag, AE Lugo. 2001. The potential for carbon sequestration of abandoned tropical agriculture and pasture lands. Restoration Ecology 8(4): 396-407.

Sokal RR, FJ Rohlf. 1995. Biometry: the principles and practice of statistics in biological sciences. $3^{\text {rd }}$ edition. New York, USA. Freeman and Company. 887 p.

Van Mantgem PJ, NL Stephenson. 2007. Apparent climatically induced increase of tree mortality rates in a temperate forest. Ecology Letters 10: 909-916.

Villalba R, HR Grau, JA Boninsegna, JC Jacoby, A Ripalta. 1998. Tree-ring evidence for long term rainfall changes in subtropical South America. International Journal of Climatology 18: 1463-1478.

Wardle DA, LR Walker, RD Bardgett. 2004. Ecosystem properties and forest decline in contrasting long-term chronosequences. Science 305: 509-513.

Zuloaga FO, O Morrone, M J Belgrano, C Marticorena, E Marchesi eds. 2008. Catálogo de las plantas vasculares del Cono Sur (Argentina, Sur de Brasil, Chile, Paraguay y Urugray). Monographs in Systematic Botany from the Missouri Botanical Garden 107: 1-3486.

Recibido: 13.05 .10

Aceptado: 12.01 .11 


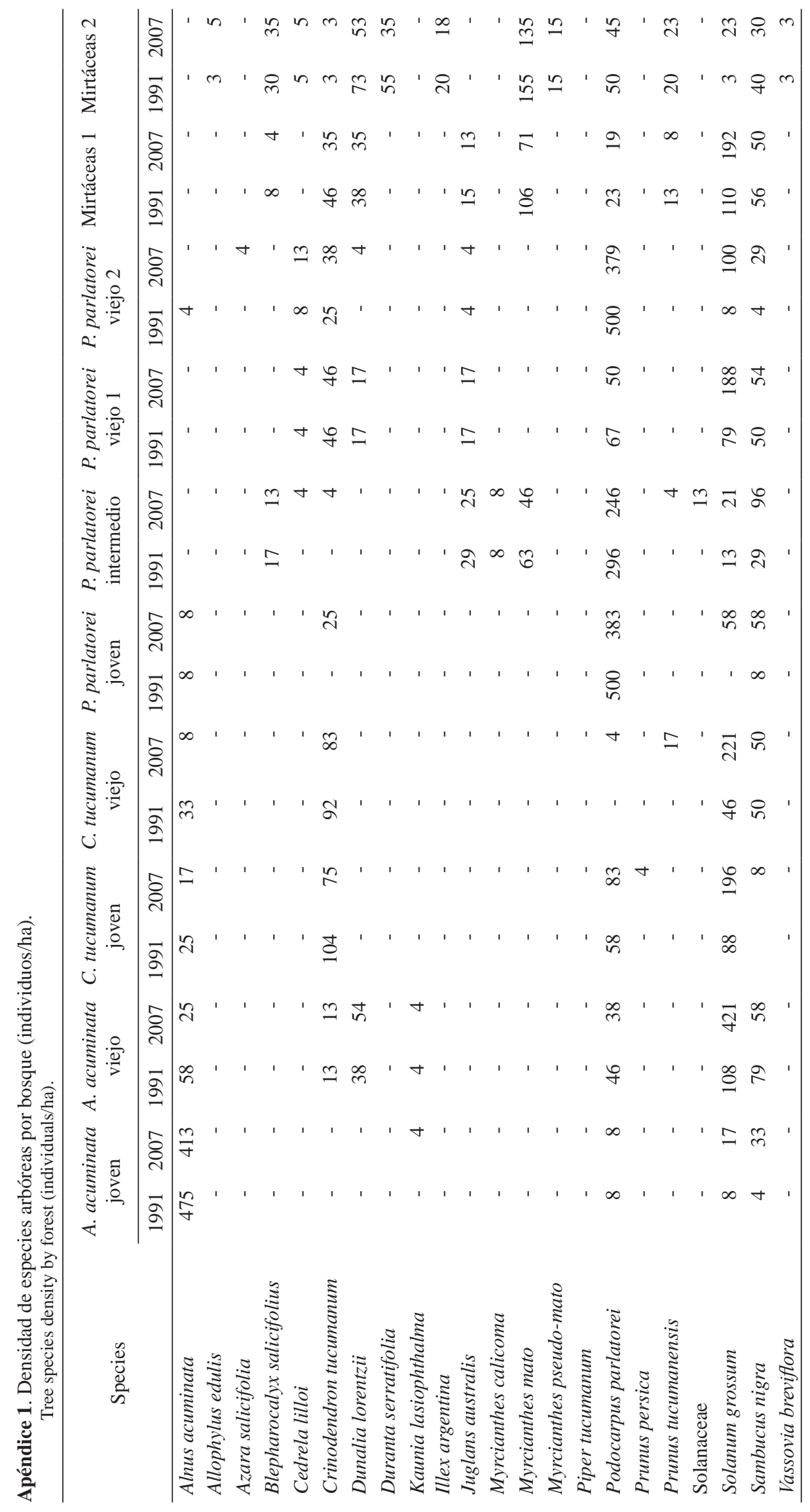


